\title{
Properties of Minimal Ghosts
}

\author{
Imants Svalbe $^{1}$ and Nicolas Normand ${ }^{1,2}$ \\ 1 School of Physics, Monash University, Melbourne, Australia \\ 2 IRCCyN, University of Nantes, Nantes, France
}

\begin{abstract}
A ghost image is an array of signed pixel values so positioned as to create zero-sums in all discrete projections taken across that image for a pre-defined set of angles. The discrete projection scheme used here is the finite Radon transform. Minimal ghosts employ just $2 N$ pixels to generate zero-sum projections at $N$ projection angles. We describe efficient methods to construct $N^{\text {th }}$ order minimal ghost images on primesized 2D arrays. Ghost images or switching components are important in discrete image reconstruction. Ghosts usually grow larger as they are constrained by more projection angles. When ghosts become too large to be added to an image, image reconstruction from projections becomes unique and exact. Ghosts can be used to synthesize image/anti-image data that will also exhibit zero-sum projections at $N$ pre-defined angles. We examine the remarkable symmetry, cross- and auto-correlation properties of minimal ghosts. The geometric properties of minimal ghost images may make them suitable to embed in data as watermarks.
\end{abstract}

\section{Introduction}

Ghosts and switching components play an important role in deciding whether an exact discrete image can be reconstructed from a given set of projections. Switching components enable the data located at certain image positions to be interchanged whilst leaving unchanged one or more projected views of that data. Similarly, ghosts are primitive images that can be added to any existing data set without changing some projected views of that data 1114. A unique image is able to be reconstructed, without prior information or assumptions, when the addition of any ghost to an image is prohibited by the constraints imposed by the projections of that data.

This paper presents an efficient way to construct very large numbers of visually distinct ghost images, each of which has $N$ zero-sum projections. These images, constructed on a $p \times p 2 \mathrm{D}$ array, where $p$ is prime, are comprised of $N$ pixels with value +1 and $N$ pixels with value -1 . Affine transforms can be applied to remould the spatial distribution of the $2 N$ pixel entries as desired.

Our first application of these minimal ghosts is to encode redundancy into image data. Ghosts form a direct, causal link between data in image space and projected views of that data in some projection space. Here we purposefully couple original image data with anti-image data built from ghost images. Combining image and anti-image data into the same space guarantees it too will have zerosum projections at the same pre-selected angles. The location of the anti-image

I. Debled-Rennesson et al. (Eds.): DGCI 2011, LNCS 6607, pp. 417 428, 2011.

(C) Springer-Verlag Berlin Heidelberg 2011 
data is restricted to fall within redundant portions of the original image space (any region where the image has constant or zero values). Redundancy that was designed in projection space is then able to be explicitly encoded into the spatial domain data. This approach has relevance to forward error-correction schemes in redundant data transmission [18].

Our second application of minimal ghosts exploits the strong geometric constraints of locating $2 N$ pixels so that they sum to zero along $N$ projection directions. Minimal ghost images have strong symmetry, compact auto-correlation and diffuse cross-correlation properties. Minimal ghost images could serve as low-visibility, high-security and robust watermarks.

Section 2 reviews briefly the discrete projection scheme in which ghost image pixel values sum to zero. Section 3 compares the complex ghost structures created by straight-forward iterated convolutions with the more demanding requirements to synthesize $N^{\text {th }}$ order ghosts using $O(N)$ rather than $O\left(2^{N}\right)$ pixels. Observations on the systematics of the minimal ghost generation method are made here. Section 4 gives examples to highlight the strong symmetry properties of minimal ghosts. In Section 5, we use minimal ghosts as the skeletal structure to construct image/anti-image combinations. The combined image/anti-image data inherits the zero-sum projection properties of the ghost image. Section 6 demonstrates the high auto- and low cross-correlations that exist between minimal ghost images of order $N$. Section 7 summarises our current conclusions and foreshadows extensions to this work.

\section{Discrete Projections, Finite Radon and Affine Transforms}

The projection scheme used here is that of the finite Radon transform (FRT) [17. FRT and its inverse are based on the paired transforms developed by Grigoryan [6] that exactly splits a 2D DFT signal into a set of $1 \mathrm{D}$ discrete oriented slices. The FRT is a highly symmetrised form of the discrete Mojette projective transform [7].

All information present in an original image $I(x, y)$ is captured, in full, by the set of discrete FRT projections, $R(t, m) . I(x, y)$ is hence exactly recoverable from $R(t, m)$. A fast algorithm to compute the FRT projections and to invert them, based on the number-theoretic transform, was presented in $[3$. The FRT is well-suited for the systematic encoding of redundant data. This approach was exploited in a proposal for an efficient forward error-correcting internet datatransmission scheme [18. Ghost images were used for systematic encoding of the data in that work.

The FRT projects data from a $p \times p$ image array, where $p$ is prime, at a set of $p+1$ discrete projection angles. Each projection is comprised of $p$ parallel translated rays. Each ray sums the intensity of exactly $p$ pixels located across the image domain. The FRT projection rays wrap periodically when they encounter any boundary of the image. The FRT, $R(t, m)$, of an image $I(x, y)$ is defined as: 


$$
\forall m \in[0, p-1], R(t, m)=\sum_{y} I\left(\langle t+m y\rangle_{p}, y\right) ; \quad R(t, p)=\sum_{x} I(x, t) .
$$

The translate parameter $t$ has $0 \leq t<p$, the projection angle parameter $m$ has $0 \leq m \leq p$. The notation $\langle j\rangle_{p}$ means taking the modulus (or integer remainder) of $j$ with respect to division by $p$.

The discrete projection angle, $m$, is defined as the gradient of a projection ray that links pixels located $m$ steps across and one pixel down from each other. The projection angle $\tan ^{-1}(1 / m)$ can be re-mapped to conventional tomographic angles, with one unique angle for each value of $m[12$. There the nearest-neighbour pixels that belong the same translate, $t$ define the ray direction. The sum $R(t, 0)$ is taken along column $t$ of the image, whilst the sum $R(t, p)$ is taken along the $t^{\text {th }}$ image row. A ghost image with $N$ zero-sum projection angles has $R(t, m)=0$ for all $t$ at $N$ values of $m$.

A scheme to perform exact, discrete rotations of image data from within the FRT domain was presented in [20. The property of parallelism is preserved by affine mappings, whether the mapping is applied in image or projection space. Arnold's cat [1] provided an early example of information-conserving, discrete image mappings. The parallel, zero-sum projected rays in any ghost image will be retained as parallel, zero-sum projections after any invertible affine transformation is applied to that image.

\section{Constructing Ghosts and Minimal Ghosts}

\subsection{An Overview of Ghosts}

Non-minimal ghosts can be constructed in a straightforward way using a process of iterated convolutions as has been shown by the earlier work of [2 5 1023]. Fig. 1a shows a pair of +1 and -1 points that will form a zero-sum projection when added to any row of an image. This primitive shape can be propagated at some new angle, for example along the column directions, as shown in fig. 1b. Adding a displaced copy of the original ghost, with signs reversed, means that the sums taken in the original direction and along the new direction are both guaranteed to be zero, as shown again in fig. 1c, where the cancellation now includes the diagonal direction. This simple propagating process results in a ghost image that contains up to $2^{N}$ pixels after displacing the accumulating shapes in $N$ directions. The complexity of iteratively-built ghost structures rises exponentially with $N$.

The displacements can be overlapped, as shown in fig. 1d, to reduce the number of pixels required to form a ghost image. In a periodic array, a large displacement that makes the image wrap, modulus p, back onto itself can also be used to effect the partial cancellation. A related paper 21] reviews the use of $N$ iterated convolutions to produce ghosts with $N$ zero-sum projection angles. That paper examines the rate of growth of the dynamic range and the increase of spatial extent of ghost images, as a function of $\mathrm{N}$, the number of zero-sum projections. 


$$
\begin{array}{ccrrrr} 
& \multicolumn{3}{c}{+1-1} & +1 & -1 \\
+1-1 & +1-1 & -1+1 & -1 & 0 & +1 \\
\text { (a) } & -1+1 & & -1+1 & +1-1
\end{array}
$$

Fig. 1. Groups of +1 and -1 pixels displaced in some new direction maintain their cancelling sums in that direction. A horizontal ghost a) is displaced vertically b) and then their union is displaced diagonally c) to produce a ghost that is invisible at three projection directions: 0, 45 and 90 degrees. d) The third dilation can be displaced so that one pixel with a +1 value has the same location as a pixel with a -1 value. This reduces the number of ghost image pixels to $2^{N}-2$.

\subsection{Minimal Ghost Construction}

Our objective here is to produce ghosts with a large number $N$ of zero-sum projections using the smallest possible number of non-zero pixels. Fewer pixels are required if $\mathrm{a}+1$ pixel can form a zero-sum with -1 pixels that lie in more than one direction. If each of the $N$ pixels with value +1 can be positioned to line up with $N$ pixels of value -1 along exactly the same $N$ directions, then a total of just $2 N$ pixels are required to construct an $N^{\text {th }}$ order ghost.

An efficient method to generate minimal ghosts was presented in 22. It relies on finding integer values $a, b, c$ and $d$ for a matrix $T=[a b ; c d]$, such that the matrix $T^{2 N}$, evaluated modulus $p$, yields the identity matrix $I=\left[\begin{array}{llll}1 & 0 ; & 0 & 1\end{array}\right]$. This procedure is akin to finding the cyclic primitive roots of a complex vector, quaternion or pseudo-tensor. Such matrices will exist for some values of $p$, because integers with exponent $N$ are cyclic when they are computed modulus $p$.

The initial matrix $T$ is used to map the $\left(x_{0}, y_{0}\right)$ coordinates of (an arbitrarily selected) +1 ghost pixel location (here called $C_{0}$ ) to produce the first -1 ghost pixel location (here called $S_{0}$ ). The matrix $T^{i}$ then remaps that current $(x, y)$ point to the location of the next +1 (for $i$ even) or -1 (for $\mathrm{i}$ odd) valued ghost pixel location (here called $C_{i}$ and $S_{i}$ ) respectively. The matrix $T^{2 N-1}$ maps the coordinates of the last $S_{i}$ point back to $C_{0}$, the coordinate of the initial starting point. Examples of $T$ and corresponding $C_{i}$ and $S_{i}$ points for some values of $N$ are given in appendix A.1. To check this procedure works, the FRT projection, $R(t, m)$, of the $p \times p$ ghost image is computed and the value of $\mathrm{R}$ must be zero, for all $t$, for $N$ values of $\mathrm{m}$.

At present, we employ a simple brute-force search method to find a candidate matrix $T$. Not all matrices $T$ for which $T^{2 N}=I(\bmod p)$ will produce minimal ghosts. We have not conducted a detailed investigation of the properties of the working matrix solutions, but have observed some of the systematic traits in the solutions for $T$ that do work. For example, $T$ has solutions when $p=2 \alpha N \pm 1$, where $\alpha$ is a positive integer. The largest value of $N$ that leads to non-trivial ghost results is $2 N=p+1$. Solutions for $N=p$ or $p-1$ can be found, but these lead to the so-called "universal ghosts", strings of pixels that form 4- or 8 -connected lines in image space. Universal ghosts form zero-sums for all but one or two of the $p+1$ FRT directions [22]. 
The structure of ghosts becomes simpler in image or projection space as $N$ approaches either 1 or $p$. This result is strikingly similar to that for the decomposition of open, median and close filters from mathematical morphology into sets of primitive erosions (or dilations) [15/16/19. In this respect, minimal ghosts with the "median" value of $N=(p \pm 1) / 2$ will have the greatest structural complexity. These median-minimal ghosts offer the optimal compromise of having a large number of zero-sum angles, yet maintaining a sparse distribution of \pm 1 pixel values spread across the image.

\subsection{Systematics of Minimal Ghost Construction}

Valid solutions for $T$ are given by $T=\left[01 ; p-1 x_{i}\right]$, where $x_{i}$ is an element of a set $X$ of integers. Each $x_{i} \in X$ gives a different, visually distinct, working ghost image solution. Variations of the above form include $T=\left[n 1 ; n x_{i}-1 x_{i}\right]$ and $T=\left[\begin{array}{lll}0 n ; n^{-1} & x_{i}\end{array}\right]$, where $n^{-1}$ is the inverse of $n$, modulus $p$. Here the common link is that $\operatorname{det}(T)=1$. The sum and product, modulus $\mathrm{p}$, of the full set of integers $x_{i} \in X$ also have interesting properties. For example, for $N=2 p^{\prime}$, where $p^{\prime}$ is prime, $\prod x_{i}= \pm p^{\prime}$ with the sign fixed by $p^{\prime}=4 \alpha \pm 1$ for some integer $\alpha$. When $N$ is even, $X$ contains pairs of complementary values, $x_{i}$ and $p-x_{i}$.

The ghost images generated by each solution $x_{i}$ appear visually distinct, but they are related to each other through affine spatial transforms. The integers $x_{i}$ depend on $N$ and the array size $p$ for which $T^{2 N}=I$. Some examples of the set $X$ that correspond to various $N$ are given in appendix A.2. However the size of the set $X, n_{X}$, depends only on the value of $N$. If $N$ is prime, $N=2 n_{X}(N)+1$; if $N$ is a power of a prime, for example $p^{\alpha}$, then $n_{X}=p^{\alpha-1} n_{X}(p)$. For composite $N$, for example, $N=i \times j \times k \ldots$, then $n_{X}(N)=(2 m-1) n_{X}(i) \times n_{X}(j) \times n_{X}(k) \ldots$, where $\mathrm{m}$ is the number of odd factors of $N$.

Choosing different initial coordinates $C_{0}$, also gives rise to visually different ghost images. Often the $N$ values of $m$ that form zero-sums will fall into distinct, non-overlapping selections of the $p+1$ possible discrete projection angles. The choice of the initial location $\left(x_{0}, y_{0}\right)$ for $C_{0}$ is arbitrary, provided only that this choice does not lead to degenerate mappings, where the coordinates for $C_{i+1}=$ $K \times C_{i}$ and $S_{i+1}=K^{\prime} \times S_{i}$, for some constants $K$ and $K^{\prime}$. Degeneracy results when the same projection angle $m$ satisfies the zero-sum constraint $N$ times.

By symmetry, if the choice $C_{0}=(j, k)$ leads to a degeneracy, then so too does $C_{0}=(k, j)$. Increased levels of degeneracy occur for the smaller integers in the set $X$. If $(i, j)$ and $(i, k)$ are degenerate choices for $C_{0}$, we find that $\langle j+k\rangle_{p}=x_{i}$. Degenerate mappings do not occur for all $N$. For example, when $N=(p+1) / 2$, exactly two sets of mutually exclusive zero-sum projection angle sets can form. There are no 'spare' projection angles to act as degenerate $m$ 's.

The size of the set $X$, its constituency and the degeneracy under the choice of array size $p$ and the $C_{0}$ location are all interesting parameters to study from a number-theoretic perspective. These parameters rely on the spacing of prime integers and cyclic permutations of prime sets. 

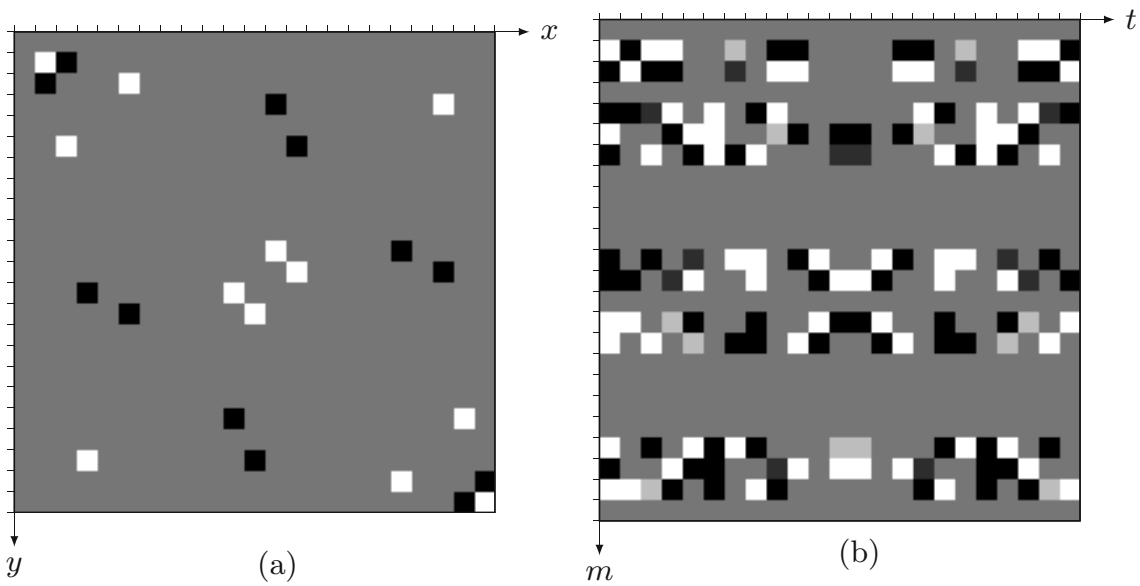

Fig. 2. a) A $23 \times 23$ image space $I(x, y)$, containing twelve +1 pixels (white) and twelve -1 pixels (black). The pixel locations are designed to make the projected sums of this image vanish at 12 pre-selected angles. b) The $23 \times 24$ discrete projections, $R(t, m)$, of the image in a). The projection translate variable, $t$, is plotted horizontally, with the discrete projection angle, $m$, shown as the vertical axis. $R(0,0)$ is at the top left corner of b). Note all the projection values at 12 distinct angles ( $m=0,3,7,8,9,10,13,16,17,18,19,23)$ have the value zero (shown here as grey).

An example $12^{\text {th }}$ order minimal ghost image is shown in fig. 2a, This $23 \times 23$ image contains 12 pixels with value +1 (shown as white) and 12 pixels with value -1 (shown as black). Projecting this image results in zero-sums at 12 projection angles, as shown in fig. 2b.

\section{Properties of Minimal Ghost Images}

The zero-sum properties of a ghost image remain invariant under intensity scaling (for example, using pixel values of $\pm g$ instead of \pm 1 ), under image translation (where addresses wrap modulus $\mathrm{p}$ ) and affine transforms where the mappings are done modulus $p$ (provided the affine mapping, as expressed in homogenous coordinates, has a proper inverse). The sum of scaled and translated $N^{\text {th }}$ order ghost images corresponding to a given set of $N$ projection angles is then also a ghost image at those same $N$ angles.

A most remarkable property of minimal ghosts is their strong symmetry. When $N$ is even, ghost images generate $N / 2$ lines that link pairs of the +1 points and $N / 2$ lines that link pairs of the -1 points. Incredibly, these $N$ lines have a common intersection point that exactly bisects each of these $N$ lines (see fig. (3)) For odd $N$ ghosts, the $N$ lines linking pairs of +1 and -1 points have a common intersection point that also bisects each of these $N$ lines. This remarkable symmetry recalls the " $n$-gon" behaviour of cyclic integers in the Fermat and Carmichael prime number sets [3]. 


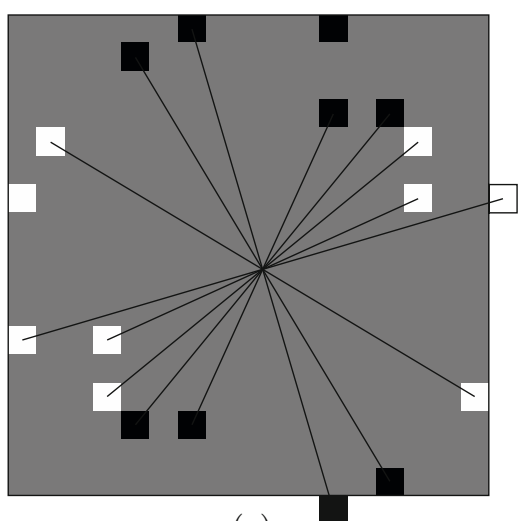

(a)

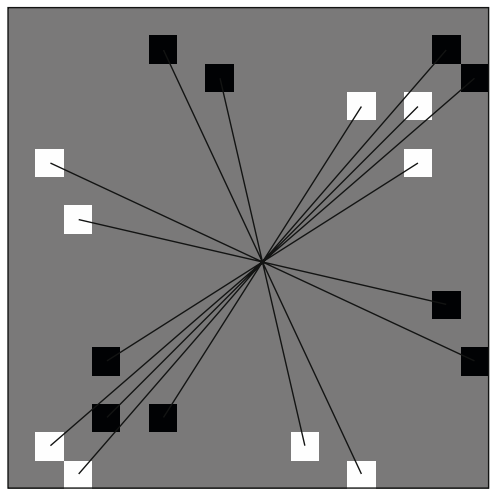

(b)

Fig. 3. Minimal ghosts for a) $N=8, x_{i}=8$ for $C_{0}=(1,4)$ and b) $N=9, x_{i}=3$ for $C_{0}=(1,5)$ on a $17 \times 17$ array. Lines joining paired ghost pixel locations are shown. The $N$ lines are all perfectly bisected at a single point of symmetry, located here at $(p / 2, p / 2)$. The position of two pixels in a) have been wrapped to the opposite edge of the array, modulus $p$ (shown by the box) to complete the symmetry.

For each non-degenerate choice of starting point, $C_{0}$, the symmetry point for a ghost image generated by the $T^{2 N}$ method is located at $(p / 2, p / 2)$. Other symmetry points, for example, $(0,0),(p / 2,0)$ and $(0, p / 2)$ are located between periodic replications of the $p \times p$ ghost image. The $N$-line bisection symmetry is preserved after applying affine transforms, but can be hard to spot visually. The mean location, $\left(x_{N}, y_{N}\right)$, of all of the $C_{i}$ points is always coincident with the mean location of all of the $S_{i}$ points. We find this point using $x_{N}=N^{-1} \sum C_{i}\left(x_{i}\right)$ and $y_{N}=N^{-1} \sum C_{i}\left(y_{i}\right)$ (or, equally, by using the $\sum S_{i}$ coordinates). Here $N \times N^{-1} \equiv 1(\bmod p)$.

The ghost images generated by the $T^{2 N}$ method always have either odd- or even-reflective symmetry about the leading diagonal. The ghost image of fig. $2 \mathrm{a}$ has even symmetry, whilst those of fig. 3 both have odd symmetry. After a transpose operation, the positions of the $C_{i}$ and $S_{i}$ pixels are exchanged $\left(C_{i} \leftrightarrow S_{i}\right)$ under odd symmetry or simply swap positions $\left(C_{i} \leftrightarrow C_{j}, S_{i} \leftrightarrow S_{j}\right)$ under even symmetry.

\section{Compacted Ghosts and the Construction of Image/Anti-image Data}

Here we warp the structure of a ghost image, like those in fig. 3, using affine transforms. The aim is to isolate a single ghost image pixel as far away as possible from its neighbouring points. This warping opens up a large space in which the targeted point can make maximum use of the ghost translation invariance property. Fig. 4a shows a $127 \times 127$ ghost image with $N=7$. It has a single +1 
point at $(0,0)$ and the next non-zero-pixel is located at $(63,33)$. The ghost in fig. 4a can be scaled to some arbitrary intensity and then translated across 127 pixel locations for each of 33 rows. The scaled and translated ghosts can be used to portray an image.

An anti-image is constructed by scaling the $N$ ghost pixels by a different intensity at each translation of the ghost image. The space that follows the clear rows of the first isolated ghost pixel then contains $N-1$ positive and $N$ negative copies of the image data. The combined image and anti-image data has zero-sums at the same $N$ selected projection angles as the ghost image. Fig. 4b shows an example of an image and anti-image constructed from a $127 \times 33$ section, cropped from the Lena image, embedded in a $127 \times 127$ space. The ghost here has order $N=7$. Both images shown in fig. 4 have zero-sum projections at $\mathrm{m}=\{5,38$, $60,68,85,102,110\}$.

The interest here lies in maximising the amount of clear image space that can be created by affine distortion of these minimal ghost images. We have, so far, only applied random affine mappings to the ghost images and then searched to find those ghosts that yield the largest clear row space. The clear-space capacity varies with $N$ and $p$. For example, at $p=421$, we can find 130 clear rows when $N=4$. Table 1 gives values for $N_{\text {rows }}$, the number of empty image rows, as a function of $N$, the number of zero-sum projections, for $p=127$.

The solutions given in Table 1 are the result of a non-exhaustive, brute-force search. We intend to apply these compacted ghosts to help symmetrise sets of Mojette projections and hence reconstruct images from asymmetric sets of projection data [4] and for data encoding schemes [18].

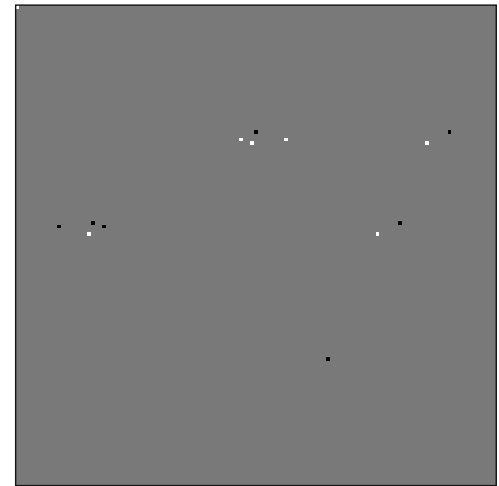

(a)

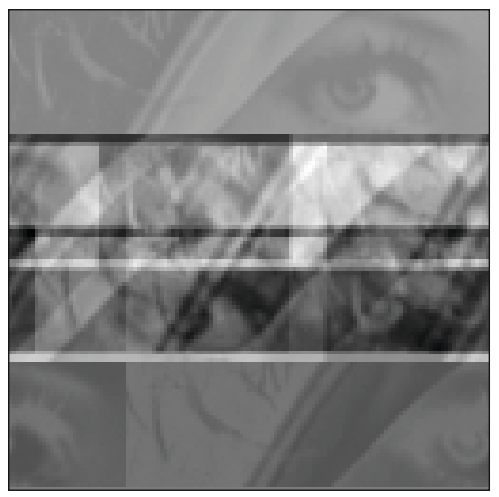

(b)

Fig. 4. a) An $N=7$ compacted ghost in a $127 \times 127$ space that exhibits a large clear space between ghost pixels. There are 33 rows of clear image space between the +1 pixel at $(0,0)$ and the next -1 ghost image pixel, located at $(63,33)$. b) A $127 \times 127$ image comprised of data (rows 0-33) followed by anti-data (rows 34-126). The FRT of the combined image shown in b) is zero at 7 discrete projection angles. 
Table 1. $N_{\text {rows }}$, the largest number of completely empty rows, for a $127 \times 127$ ghost image space, as a function of $N$, the number of zero-sum angles

$$
\begin{array}{rrrrrrrrrrrrr}
N & 3 & 4 & 6 & 7 & 8 & 9 & 14 & 16 & 18 & 21 & 32 & 64 \\
N_{\text {rows }} & 35 & 33 & 22 & 32 & 30 & 20 & 15 & 14 & 13 & 9 & 7 & 5
\end{array}
$$

\section{2D Correlations between Minimal Ghost Images}

There is considerable interest in the generation of $2 \mathrm{D}$ sequences that have perfector near-perfect correlation properties 8913. A perfect sequence on an $m \times n$ array has a normalised peak auto-correlation value of $m \times n$ at one location and is zero everywhere else. Near-perfect sequences have off-centre correlation values of \pm 1 or \pm 2 , rather than zero. The theoretical interest here extends to practical applications in encryption, message-coding and watermarking applications [13].

The remarkable geometric properties of minimal ghosts lead us to examine the correlation properties of ghost images. Ghosts with $N$ zero-sums contain $N$ pixels of +1 and $N$ pixels of -1 . The peak value of a $p \times p$ ghost image auto-correlation is then $2 \mathrm{~N}$, considerably less than $p^{2}$.

However these ghost images are sparse and "random" in appearance and so are easy to hide as small perturbations of existing data structures. The linear structure of universal ghosts, where $N \approx p$, makes them less suitable as candidates for watermarks. The largest non-trivial (median) ghost images contain $N=(p+1) / 2$ points. These ghosts provide randomised, high density coverage

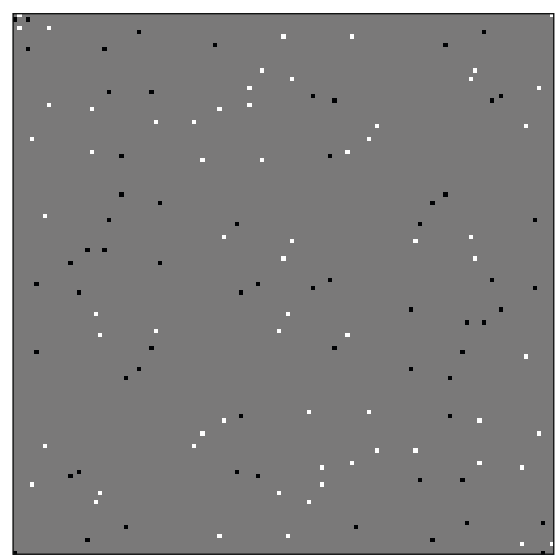

(a)

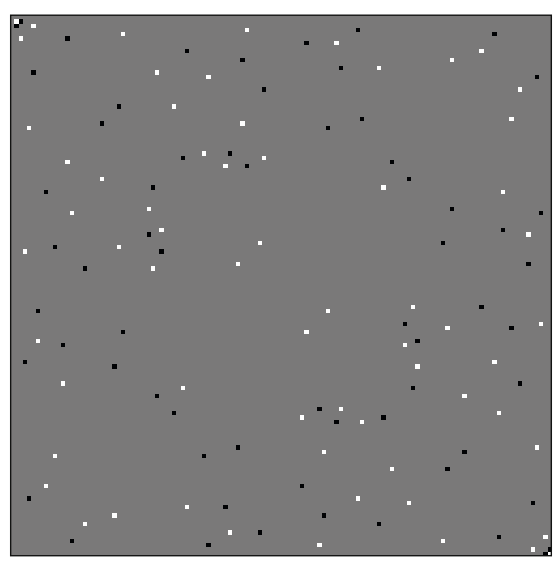

(b)

Fig. 5. Ghost images for $N=64, p=127$ generated by $x_{i}=3$ and a) $C_{0}=(1,0)$ and b) for $C_{0}=(1,1)$. The auto-correlation of a) has a peak value of 128 at $(127,127)$, other locations have absolute values $\leq 2$. The cross-correlation between a) and b) has values $+1,0$ or -1 . 
of the $p \times p$ array space and have a peak auto-correlation value of $\mathrm{p}+1$. Although ghost images are generated on $p \times p$ arrays, the ghost images can be regarded as occupying a subset of this space, particularly after applying the compacting affine transforms discussed in section 5 .

The strength of the cross-correlation between minimal ghost images depends on whether they have the same value of $N$, if the ghosts were generated by the same matrix $T$ with the same $x_{i} \in X$, and if they have the same set of zero-sum angles (i.e. depending on the choice of $C_{0}$ ). The worst case cross-correlations between ghost images give rise to values of \pm 4 or less. The cross-correlation of a ghost with a random affine-mapped version of itself is thus restricted to \pm 4 or less, independently of the array size p.

The off-peak value of ghost image auto-correlations is \pm 2 or less. For example, at $p=127$ and $N=64$, the ghost generated by $x_{i}=3$ has a peak autocorrelation value of 128 , with 3844 pixels of grey value $-2,504$ at $-1,55316$ at zero, 524 at +1 and 3720 pixels with value +2 (see fig. [5).

\section{Conclusions}

An $N^{\text {th }}$ order ghost image contains pixels with value \pm 1 or 0 that are aligned to create zero-sum projections for $N$ discrete projection angles. We have demonstrated the means to generate large numbers of distinct ghost images on $2 \mathrm{D}$ prime-sized arrays, with each image containing the minimum possible $2 N$ nonzero pixels.

These minimal ghost images exhibit interesting discrete geometric properties. The $N$ lines that link specific pairs of ghost pixels were shown to intersect at a single symmetry point that bisects, exactly, each of these $N$ lines. The shape of ghost images can be warped by affine transforms to maximise or minimize the distance between neighbouring ghost pixels. Compact ghost images permit the construction of significantly-sized blocks of image/anti-image data that inherits zero-sum projection properties from the parent ghost image.

The auto-correlation of an $N^{\text {th }}$ order minimal ghost has a peak value of $2 N$, with side lobes of \pm 2 or less. The peak cross-correlation value for pairs of minimal $N^{\text {th }}$ order ghosts can be as low as \pm 1 for ghosts that have distinct sets of zero-sum angles, but it is always less than \pm 4 . Many ghost images can be nested within the same data region with zero or minimal pixel overlap. The correlation properties of nested and co-located ghost images suggest very flexible and reliable signal recovery strategies. Minimal ghost images may make good data watermarks.

We are keen to further understand the properties of the matrix $T$ and the set $X$ of generating integers that produce ghosts on prime-sized arrays. Using a symbolic mathematics package to explore the elements of the matrices $T$ and $T^{i}$ has revealed some interesting polynomial patterns. There are strong links between $N^{\text {th }}$ order minimal ghost images and $N$ by $N$ Latin squares [22]. Large order Latin squares provide very interesting data encryption prospects.

It would also be useful to develop efficient methods to compact ghost images by purposeful means, rather than, as now, searching through large numbers of 
random affine ghost transformations. Finding the maximum and minimum size of the empty space between non-zero ghost pixels is also important. Establishment of a theoretical upper-bound for "collision-free" translation of an $N^{\text {th }}$ order ghost in a $p \times p$ image would be an important finding.

The method used here to find minimal ghosts on prime $2 \mathrm{D}$ arrays can probably be extended to obtain $N^{\text {th }}$ order zero-sums projected across planes or lines in prime $3 \mathrm{D}$ cubes and higher dimension arrays. This will also be an interesting topic to pursue.

Acknowledgement. Nicolas Normand worked, from June 2009 to July 2010, in the School of Physics at Monash University, and acknowledges the support of an International Fellowship, granted through the Australian Research Council (LX0989907). Imants Svalbe acknowledges ongoing support for this work from the School of Physics and from the Faculty of Science at Monash University.

\section{References}

1. Arnold, V.I., Avez, A.: Problèmes ergodiques de la mécanique classique. GauthierVillars, Paris (1967); English translation: Benjamin, New York (1968)

2. Barcucci, E., Del Lungo, A., Nivat, M., Pinzani, R.: X-rays characterizing some classes of discrete sets. Linear Algebra and its Appl. 339(1-3), 3-21 (2001)

3. Chandra, S., Svalbe, I.D.: A fast number theoretic finite Radon transform. In: DICTA 2009, pp. 361-368. IEEE, Melbourne (2009)

4. Chandra, S., Svalbe, I.D., Guédon, J.-P.: An exact, non-iterative mojette inversion technique utilising ghosts. In: Coeurjolly, D., Sivignon, I., Tougne, L., Dupont, F. (eds.) DGCI 2008. LNCS, vol. 4992, pp. 401-412. Springer, Heidelberg (2008)

5. Gardner, R.J., Gritzmann, P.: Discrete tomography: determination of finite sets by X-rays. Trans. AMS 349(6), 2271-2295 (1997)

6. Grigoryan, A.M.: New algorithms for calculating discrete Fourier transforms. Zhurnal Vychislitel'noi Matematiki i Matematicheskoi Fiziki 26(5), 1407-1412 (1986)

7. Guédon, J.P. (ed.): The Mojette Transform: Theory and Applications. Wiley-ISTE, Chichester (2009)

8. Hariharan, R.: Near perfect sequences of odd length. In: Int. Workshop on Signal Design and its Appl. in Comm., pp. 4-7. IEEE, Fukuoka (2009)

9. Hayashi, T.: Zero-correlation zone sequence set construction using an even-perfect sequence and an odd-perfect sequence. IEICE Trans. on Fundamentals of Electronics, Comm. and Computer Sciences 90-A(9), 1871-1875 (2007)

10. Herman, G.T., Davidi, R.: Image reconstruction from a small number of projections. Inverse Problems 24(4) (2008)

11. Katz, M.B.: Questions of uniqueness and resolution in reconstruction from projections. Lecture Notes in Biomath., vol. 26. Springer, Berlin (1978)

12. Kingston, A.M., Svalbe, I.D.: Projective transforms on periodic discrete image arrays. In: Adv. in Imaging and Electron Physics, vol. 139, pp. 75-177. Elsevier, Amsterdam (2006)

13. Kuznetsov, O.: Perfect sequences over the real quaternions. In: Int. Workshop on Signal Design and its Appl. in Comm., pp. 8-11. IEEE, Fukuoka (2009)

14. Louis, A.K.: Picture reconstruction from projections in restricted range. Mathematical Methods in the Applied Sciences 2, 209-220 (1980) 
15. Maragos, P., Schafer, R.W.: Morphological filters-parts I \& II. In: Proceedings of IEEE Int. Conf. on Acoustics, Speech, and Signal Processing (ICASSP 2002), vol. 35, pp. 1153-1184. IEEE, Los Alamitos (1987)

16. Matheron, G.: Random Sets and Integral Geometry. Wiley, New York (1975)

17. Matúš, F., Flusser, J.: Image representation via a finite Radon transform. IEEE Trans. on Pattern Analysis and Machine Intelligence 15(10), 996-1006 (1993)

18. Normand, N., Svalbe, I.D., Parrein, B., Kingston, A.M.: Erasure coding with the finite Radon transform. In: IEEE Wireless Comm. \& Networking Conf., Sydney, pp. 1-6 (April 2010)

19. Serra, J.: Image analysis and mathematical morphology. In: Theoretical Adv., vol. 2. Academic Press, London (1982)

20. Svalbe, I.D.: Exact, scaled image rotations in finite Radon transform space. Pattern Recognition Letters (2010) (in press)

21. Svalbe, I.D., Chandra, S.: Growth of discrete projection ghosts created by iterated dilations. In: DGCI 2011. LNCS, Springer, Nancy (2011)

22. Svalbe, I.D., Normand, N., Nazareth, N., Chandra, S.: On constructing minimal ghosts. In: DICTA 2010, Sydney, Australia (December 2010)

23. Zopf, S.: Construction of switching components. In: Nyström, I., Sanniti di Baja, G., Svensson, S. (eds.) DGCI 2003. LNCS, vol. 2886. Springer, Heidelberg (2003)

\section{A Appendices}

\section{A.1}

Example lists of $C_{i}$ and $S_{i}$ ghost pixel coordinates and their zero-sum gradients, $m_{i}$ are given below for (left) $p=17, N=8, T=[10 ; 168], C_{0}=(1,4)$, see fig. $3 \mathrm{a}$ and (right) $p=17, N=9, T=\left[\begin{array}{lll}1 & 0 ; 16 & 3\end{array}\right], C_{0}=(1,5)$, see fig. $3 \mathrm{~b}$,

$$
\begin{aligned}
& C_{i}=\begin{array}{rrrrrrrrr}
1 & 14 & 0 & 3 & 16 & 3 & 0 & 14 \\
4 & 6 & 11 & 13 & 13 & 11 & 6 & 4
\end{array} \quad C_{i}=\begin{array}{rrrrrrrrr}
1 & 14 & 12 & 2 & 2 & 12 & 14 & 1 & 10 \\
5 & 3 & 16 & 7 & 16 & 3 & 5 & 15 & 15
\end{array}
\end{aligned}
$$

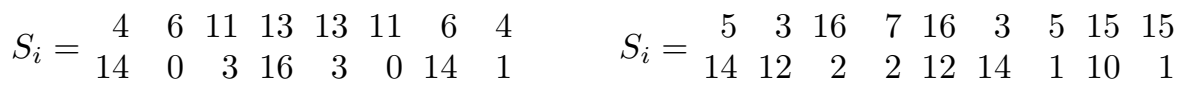

$$
\begin{aligned}
& m_{i}=\begin{array}{lllllllll}
1 & 2 & 3 & 5 & 6 & 7 & 9 & 16 & m_{i}=
\end{array} \begin{array}{lllllllll}
4 & 5 & 7 & 8 & 10 & 12 & 13 & 15 & 16
\end{array}
\end{aligned}
$$

\section{A.2}

Lists of $x_{i} \in X$, for some example values of $N$, at selected values of $p$ are given below.

$$
\begin{aligned}
& N=7, \quad p=13:\{356\} \\
& N=8, p=17:\left\{\begin{array}{lll}
58912\} & p=127:\{42487985\}
\end{array}\right. \\
& N=9, p=17:\left\{\begin{array}{lll}
3410\} & p=127:\{5387114\}
\end{array}\right. \\
& N=11, p=23:\left\{\begin{array}{llll}
9 & 12131719\} & p=131:\{1033537582\}
\end{array}\right. \\
& N=14, p=29:\{101213161719\} p=139:\{3746548593102\} \\
& N=37, p=73:\{358111820262729373840435051565766\} \\
& N=39, p=79:\{52632353741555658597276\}
\end{aligned}
$$

